Editorial

\title{
Novel Advances in the Development of Machine Learning Solutions for Scientific Programming
}

\author{
Vicente García-Díaz $\mathbb{D}^{1},{ }^{1}$ Edward R. Núñez-Valdez $\mathbb{D}^{1},{ }^{1}$ Vijender K. Solanki, ${ }^{2}$ \\ and Carlos E. Montenegro-Marin ${ }^{3}$ \\ ${ }^{1}$ University of Oviedo, Oviedo, Spain \\ ${ }^{2}$ CMR Institute of Technology, Hyderabad, India \\ ${ }^{3}$ Universidad Distrital Francisco José de Caldas, Bogotá, Colombia
}

Correspondence should be addressed to Vicente García-Díaz; garciavicente@uniovi.es

Received 26 May 2019; Accepted 26 May 2019; Published 1 July 2019

Copyright (c) 2019 Vicente García-Díaz et al. This is an open access article distributed under the Creative Commons Attribution License, which permits unrestricted use, distribution, and reproduction in any medium, provided the original work is properly cited.

Scientific programming is a multidisciplinary field that uses advanced methods to understand and solve complex problems. Meanwhile, machine learning is the field that uses statistical techniques to give computer systems the ability to learn and extract knowledge from data, answering questions, and solving problems in various application domains, without the need of being explicitly programmed. Both are exciting, complex, and interrelated fields in which advances are taking place at a great pace.

This special issue aims to bring together some of the most important advances related to the union of scientific programming and progresses in the field of machine learning. Thus, after a rigorous selection process, this number includes 9 works that allow us to continue exploring the possibilities that machine learning can provide, particularly for scientific programming.

The first paper is entitled "A 64-Line Lidar-Based Road Obstacle Sensing Algorithm for Intelligent Vehicles" by $\mathrm{H}$. Wang et al. In this work, the authors present a novel approach for road obstacle sensing through an effective and real-time-based algorithm. This work provides contributions to improve the safety of drivers using 64-line lidar sensors and classifiers based on support vector machines. The focus is on detecting obstacles with clustered object positions and specific features.

The second paper is entitled "A Low Cost Named Entity Recognition Research Based on Active Learning" by $\mathrm{H}$. Huang et al. In this case, the authors propose advances in the field of natural language processing. They focus on named entity recognition by using active learning together with the conditional random field classifier, which serves to improve its performance. To this end, the authors apply clusters created with the $K$-means method. The testing data include Chinese judicial documents and Chinese electronic medical records.

The third paper is entitled "Design and Implementation of a Machine Learning-Based Authorship Identification Model" by W. Anwar et al. The paper focuses on authorship identification in English and Urdu languages using a latent Dirichlet allocation model. The presented approach is an unsupervised computational methodology that can handle the heterogeneity of datasets, diversity in writing, and the inherent ambiguity of the Urdu language. Finally, the authors used a big corpus to test the performance of the presented approach.

The fourth paper is entitled "A non-disturbing service to automatically customize notification sending using implicitfeedback" by F. López Hernández et al. The work addresses the problem of automatically customizing notifications in a nondisturbing way. The idea is to use implicit feedback in order not to ask preferences directly to users. To that end, a hybrid filter that combines both content and collaborative filtering to predict the best notifications for users in each concrete situation is also presented.

The fifth paper is entitled "Consensus ClusteringBased Undersampling Approach to Imbalanced Learning" by A. Onan. In this work, the author works on class imbalance, which is one important issue in machine learning. He presents a consensus clustering-based undersampling approach to imbalanced learning. The 
work also shows an empirical analysis with 33 small-scale and 2 large-scale imbalanced classification benchmarks together with 5 clustering algorithms and their combinations, also with supervised learning methods.

The sixth paper is entitled "Towards the Construction of a User Unique Authentication Mechanism on LMS Platforms through Model-Driven Engineering (MDE)" by J. F. HerreraCubides et al. In this case, the authors work on authentication issues. They propose a security abstraction model on learning management systems based on model-driven engineering. To that end, the authors presented a metamodel with a set of guidelines on how to carry out authentication considering the different involved stakeholders.

The seventh paper is entitled "Design and Development of a Large Cross-Lingual Plagiarism Corpus for Urdu-English Language Pair" by I. Haneef et al. In this work, the authors focus on cross-lingual plagiarism that detects plagiarism of a text even if it is written in different languages by checking semantic similarities. The paper is intended to present the results working with English and Urdu languages. To meet the goals, the authors have created a corpus with 2398 source pairs, which is publicly available for research purposes.

The eighth paper is entitled "Avionics Graphics Hardware Performance Prediction with Machine Learning" by S. R. Girard et al. The authors create a system design tool to help predict the rendering performance of graphical hardware based on the OpenGL library. That is, they propose to replace expensive alternatives by a predictive software application running on a desktop computer. In addition, the authors render an industrial scene with features not used during the training phase, getting an error of less than 4 frames per second.

The last paper is entitled "Effects of challenging weather and illumination on learning-based License Plate Detection in non-controlled environments" by A. Rio-Alvarez et al. The authors work on automatic license plate recognition systems focusing on license plate detention. They study the differences in the effectiveness of different descriptors for concrete weather conditions. They state that images affected by raining should not be included in training sets, but images affected by low illumination conditions should be included because they increase performance.

\section{Conflicts of Interest}

The authors declare that they have no conflicts of interest.

\section{Acknowledgments}

The guest editors would like to thank all the authors for contributing their efforts and all the reviewers for their time and rigorous reviews, making this special issue possible.

Vicente García-Díaz Edward R. Núñez-Valdez Vijender K. Solanki

Carlos E. Montenegro-Marin 


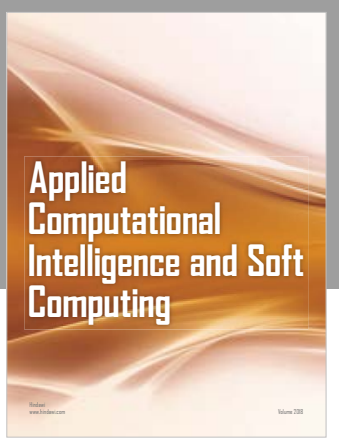

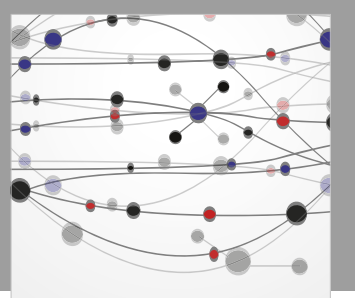

The Scientific World Journal
Submit your manuscripts at

Computing
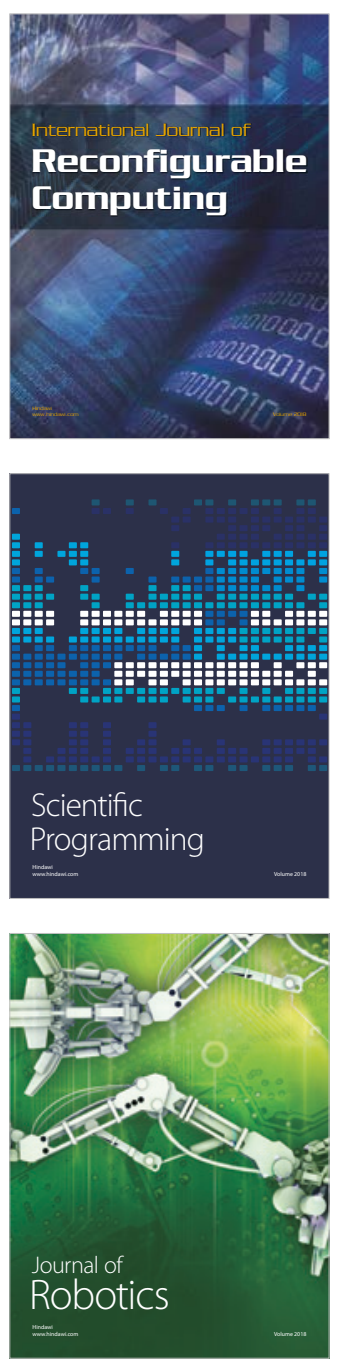

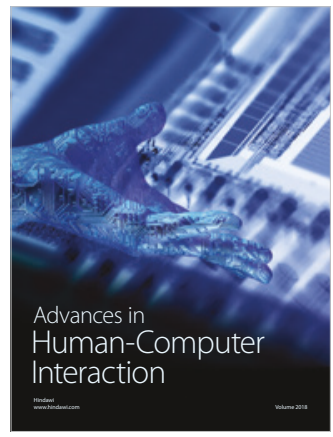

Human-Compute

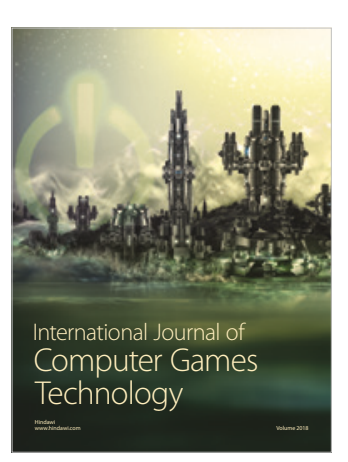

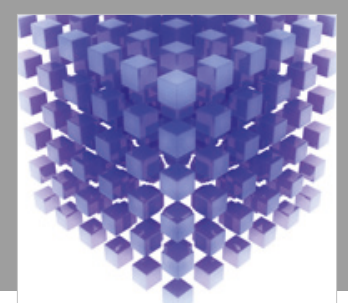

Mathematical Problems in Engineering

\section{Engincering}
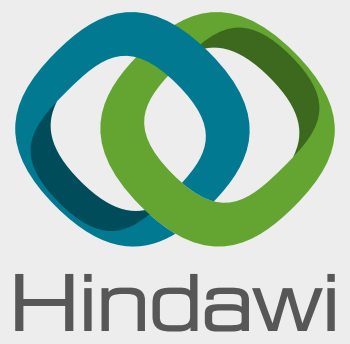

www.hindawi.com
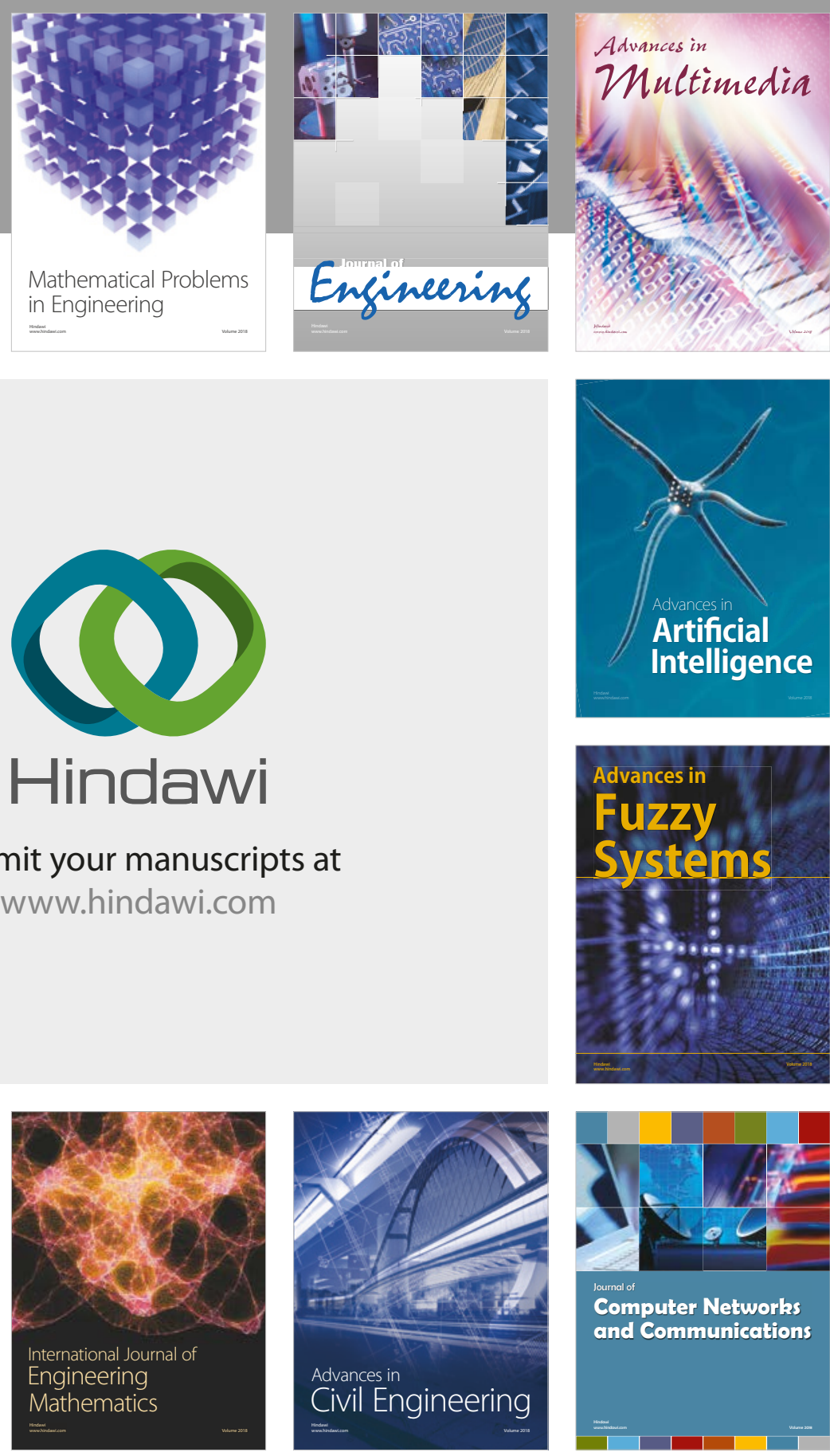

Computer Networks and Communications

Multimedia
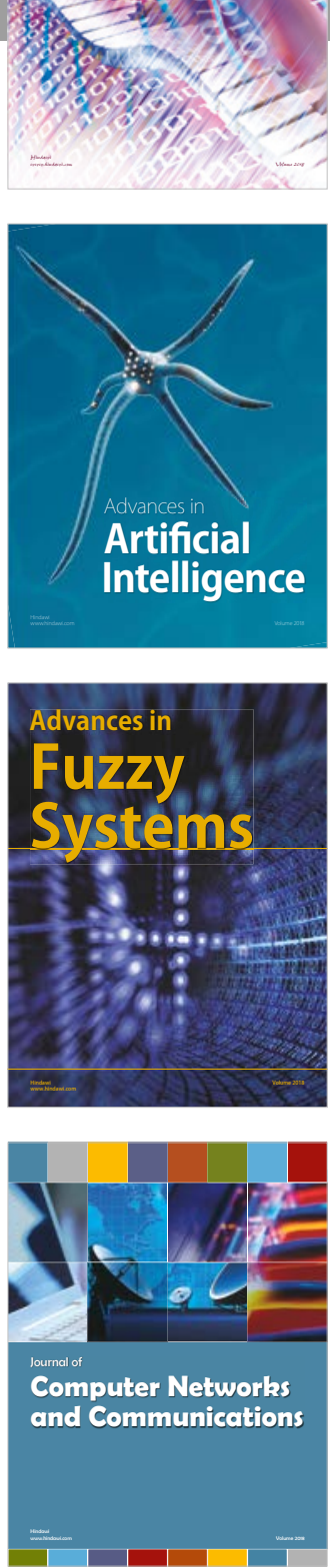

Advances in

Modelling \&

Simulation

in Engineering

interaction

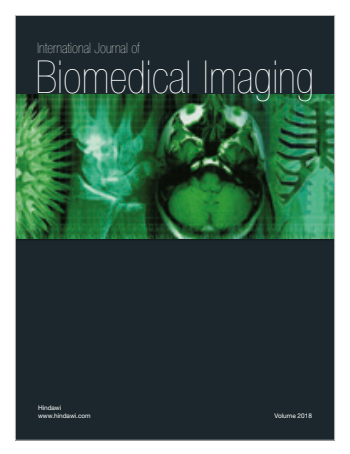

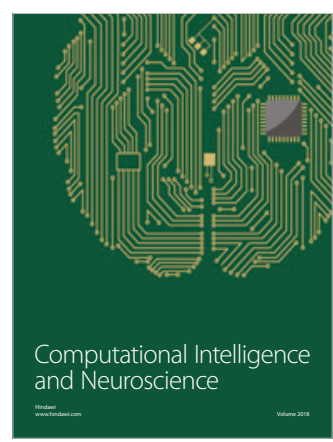

